Review began 01/21/2022 Review ended 01/31/2022 Published 02/06/2022

๑) Copyright 2022

Takahashi et al. This is an open access article distributed under the terms of the Creative Commons Attribution License CCBY 4.0., which permits unrestricted use, distribution, and reproduction in any medium, provided the original author and source are credited.

\section{Optic Neuropathy Caused by a Perineural Sarcoid Lesion}

\author{
Yasuhiro Takahashi $^{1}$, Shinjiro Kono ${ }^{1}$, Aric Vaidya ${ }^{1,2}$, Hirohiko Kakizaki ${ }^{1}$ \\ 1. Oculoplastic, Orbital and Lacrimal Surgery, Aichi Medical University Hospital, Nagakute, JPN 2. Oculoplastic, \\ Orbital and Lacrimal Surgery, Kirtipur Eye Hospital, Kathmandu, NPL
}

Corresponding author: Yasuhiro Takahashi, yasuhiro_tak@yahoo.co.jp

\begin{abstract}
A 74-year-old woman had a six-month history of decreased vision in the left eye. On the first examination, her left best-corrected visual acuity was 0.02 , and Goldmann visual field test revealed a central scotoma in the left eye. Magnetic resonance imaging demonstrated lesions around the optic nerve on both sides and enlargement of the lacrimal gland and superior rectus/levator palpebrae superioris muscles on both sides and the medial and inferior recti muscles on the left side. Systemic computed tomography revealed bilaterally enlarged mediastinal and supraclavicular lymph nodes. The blood test results included an elevated soluble interleukin-2 receptor. Pathological examination of the specimens harvested from the lacrimal gland on both sides, left levator palpebrae superioris muscle, and the lesion around the optic nerve on the left side showed lymphocytic infiltration with noncaseating epithelioid granuloma. After the biopsy, the patient underwent two cycles of steroid pulse therapy, followed by oral prednisolone. Although the lesions were reduced after steroid treatment, the left vision did not recover.
\end{abstract}

Categories: Ophthalmology

Keywords: vision loss, steroid, optic neuropathy, perineural mass, sarcoidosis

\section{Introduction}

Sarcoidosis involves the ocular and periocular tissues in $10 \%-60 \%$ of cases [1-4]. The most common manifestation of ocular sarcoidosis is uveitis, while extraocular lesions are relatively rare [1-6]. Involvement of the lacrimal gland is most frequent, followed by the orbit, eyelid, and lacrimal sac [1], while the optic nerve is rarely involved [2].

The neuro-ophthalmic sarcoidosis is the second frequent type in neurosarcoidosis and is found in $1 \%-5 \%$ of cases with sarcoidosis $[3,7,8]$. Most cases show optic perineuritis, while other manifestations including optic nerve compression by an orbital mass, optic nerve granulomas, and papilloedema by elevated intracranial pressure are rare [7,9-11]. We report a case of optic neuropathy secondary to a perineural sarcoid mass.

\section{Case Presentation}

A 74-year-old woman complained of decreased vision six months before referral to us. The patient had followed up at an eye clinic for cataract in both eyes, but she searched another clinic for further examination of gradually decreased vision on her own accord. Multiple orbital masses and lung and breast lesions were detected at the second clinic. She did not have history of any systemic disease or family history.

On the first examination, her best-corrected visual acuity was 1.0 in the right eye and 0.02 in the left eye. Intraocular pressure was $13 \mathrm{mmHg}$ in both eyes. Slit-lamp examination did not show cells in the anterior chamber, iris nodule, or peripheral angle synechiae. Funduscopic examination revealed a pale optic disc and mild retinal phlebitis in the left eye (Figure 1A). Relative afferent pupillary defect (RAPD) was positive in the left eye. Goldmann visual field test revealed a central scotoma in the left eye. Abduction and supraduction were restricted in the left eye. The left eye had $1.5 \mathrm{~mm}$ proptosis as compared to the right eye (Figure $1 B$ ). The lacrimal gland was palpable in both sides. The right upper eyelid was ptotic, and marginal reflex distance 1 was $0.5 \mathrm{~mm}$ on the right side and $3.5 \mathrm{~mm}$ on the left side (Figure $1 B$ ). Magnetic resonance imaging (MRI) demonstrated lesions around the optic nerve on both sides and enlargement of the lacrimal gland and superior rectus/levator palpebrae superioris muscles on both sides and the left medial and inferior recti muscles (Figures $1 \mathrm{C}, 1 \mathrm{D}$ ). The lesions showed isointensity to muscle on T1- and T2-weighted MRI images. Enhanced T1-weighted axial MRI showed the "tram-track" sign on the left side (Figure 1C). Systemic computed tomography revealed a mass in the right breast and bilaterally enlarged mediastinal and supraclavicular lymph nodes (Figure $1 E$ ). The blood test results included an elevated soluble interleukin-2 receptor (1,153 U/mL; normal range, 122-496 U/mL) and antinuclear antibody (1:40; normal range, < 1:40). Thyroid autoantibodies, rheumatoid factor, Sjögren syndrome (SS)-A and SS-B antibodies, immunoglobulin G4, anti-DNA antibody, proteinase 3- and myeloperoxidase-antineutrophil cytoplasmic antibodies, and angiotensin-converting enzyme were within the normal ranges. The patient was consulted with a breast surgeon, but the doctor judged no necessity for further examination of the breast lesion. 


\section{Cureus}

An incisional biopsy was performed under general anesthesia by two of the authors (YT and SK). The lesions in the lacrimal gland on both sides and levator palpebrae superioris muscle on the left side were partially excised via an eyelid crease approach. Next, the lesion around the left optic nerve was biopsied via a transconjunctival approach of the lower eyelid. After the surgery, the patient underwent two cycles of steroid pulse therapy. Pathological examination of the harvested specimens showed lymphocytic infiltration with noncaseating epithelioid granuloma (Figure $1 \mathrm{~F}$ ). A pulmonologist diagnosed it as a pulmonary sarcoidosis based on the findings of enlarged mediastinal lymph nodes and elevated serum interleukin-2 receptor, while the cardiologist and dermatologist ruled out the presence of cardiac and cutaneous sarcoidosis, respectively.

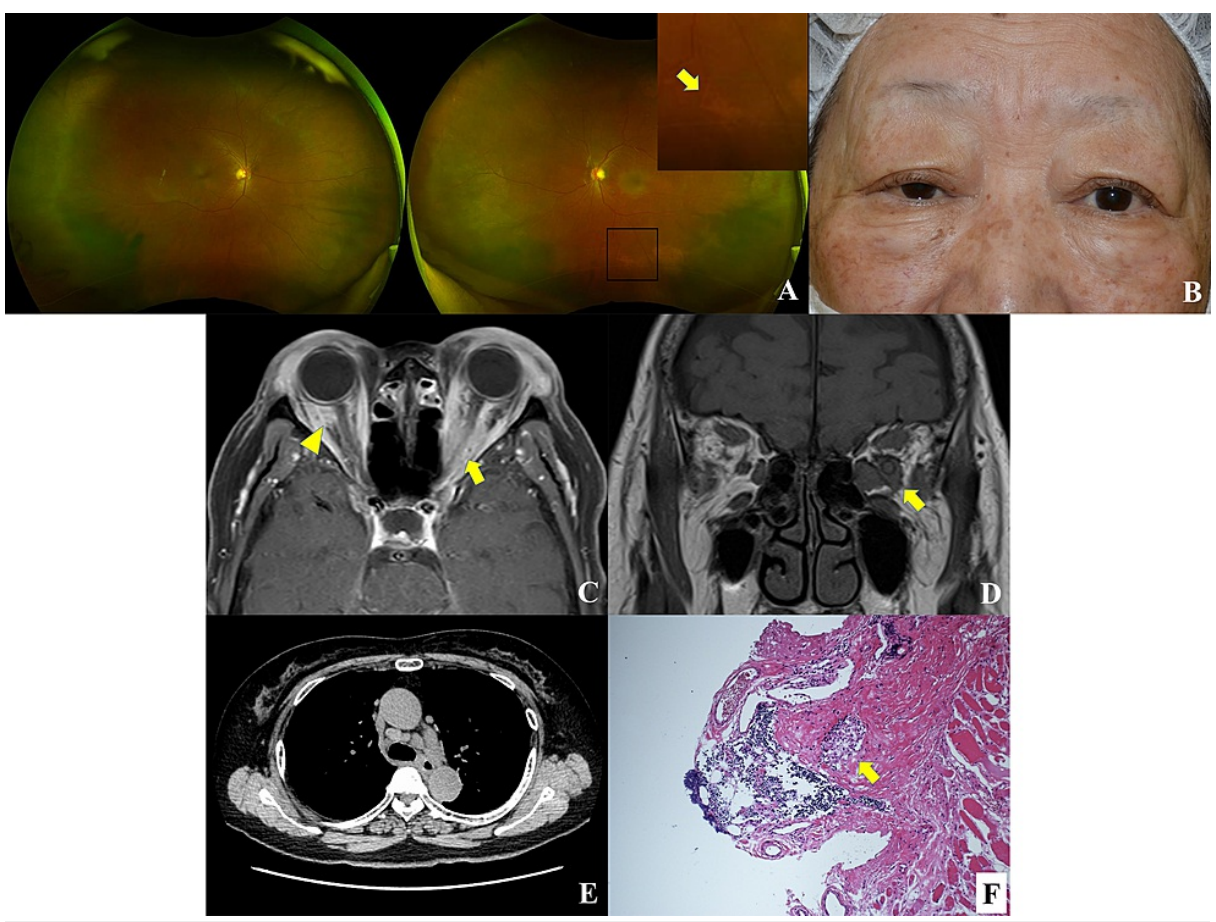

\section{FIGURE 1: Case presentation.}

A. A left fundus photo showing a pale disc and mild retinal phlebitis (arrow). B. A face photo showing proptosis on the left side and ptosis on the right side. C, D. An enhanced T1-weighted axial (C) and T1-weighted coronal magnetic resonance images (MRI) (D) showing lesions around the optic nerve on both sides (arrowhead and arrow) with the "tram-track" sign on the left side (arrow) and enlargement of the bilateral lacrimal glands, bilateral superior rectus/levator palpebrae superioris muscles, and left medial and inferior recti muscles. E. An axial chest computed tomographic images showing enlarged mediastinal lymph nodes. F. Pathological examination showing lymphocytic infiltration with noncaseating epithelioid granuloma (arrow; hematoxylin and eosin staining; magnification, $\times 100$ )

After completing the steroid pulse therapy, the patient started taking oral prednisolone and tapered it from $30 \mathrm{mg} /$ day over a period of six months. Following steroid treatment, although the lesions were reduced in size, the left vision did not recover. Ptosis on the left side developed after the biopsy, and margin reflex distance 1 was $-2.0 \mathrm{~mm}$ on the left side.

This case report adheres to the tenets of the Declaration of Helsinki as amended in 2008. Written informed consent for publication of patient record and identifiable patient face photos was obtained from the patient.

\section{Discussion}

We present a rare case of optic neuropathy secondary to a perineural sarcoid mass. Mixed results from previous studies demonstrated only four cases of optic nerve involvement and the other two cases with positive RAPD among 137 cases with orbital sarcoidosis [1,2,4,6]. Additionally, there had been a few case reports on orbital sarcoidosis with optic nerve involvement $[7,10,11]$.

The histopathological findings of the specimens harvested from the orbits corresponded to the histological diagnostic criteria of sarcoidosis proposed by the Japan Society of Sarcoidosis and Other Granulomatous Disorders in 2015 [12]. Furthermore, bilateral enlargement of the mediastinal and supraclavicular lymph nodes and elevated soluble interleukin-2 receptor fulfilled the clinical diagnostic criteria of sarcoidosis [12].

Sarcoidosis is considered a "great mimicker". A previous report showed a case of a sarcoid mass around the optic nerve presenting with "tram-track" sign, similar to optic nerve meningioma [11]. Our case had similar 
findings including perineural mass and the "tram-track" sign on enhanced axial MRI. However, bilateral lesions and other accompanying orbital lesions are rare in cases with optic nerve sheath meningioma [13]. Other differential diagnoses include metastatic tumors, leukemia, lymphoma, and other orbital inflammatory diseases $[3,14]$. However, it may be possible to differentiate these diseases, based on the results of blood tests and systemic imaging studies.

Treatment modalities for orbital sarcoidosis include intralesional, oral, and intravenous steroids, methotrexate, cyclosporine, cyclophosphamide, mycophenolate, interferon, azathioprine, hydroxychloroquine, and surgical debulking $[1-3,6,14]$. However, intravenous steroids, which were given in the present case, may be a better option for cases with optic nerve involvement [14].

The vision recovered after steroid treatment in previously reported cases with optic nerve involvement $[7,10]$. However, in our case, there was no recovery of left vision even after steroid treatment. Those previously reported cases had optic nerve edema before starting steroid treatment, while in our case, the optic nerve disc was already pale. This may be a cause of the different clinical course between the present and the previously reported cases.

\section{Conclusions}

In conclusion, we report a rare case of optic neuropathy caused by a perineural sarcoid mass. The findings of our case fulfilled both the histological and clinical diagnostic criteria of sarcoidosis. Preoperative blood tests and orbital and systemic imaging studies are essential for the definite diagnosis of neuro-ophthalmologic sarcoidosis.

\section{Additional Information \\ Disclosures}

Human subjects: Consent was obtained or waived by all participants in this study. Aichi Medical University Hospital issued approval None. We asked the institutional review board of Aichi Medical University Hospital and confirmed that the ethics approval for this report was not necessary on the basis of the ethical guidelines for medical and health research involving human subjects established by the Japanese Ministry of Education, Culture, Sports, Science, and Technology and the Ministry of Health, Labour, and Welfare. Conflicts of interest: In compliance with the ICMJE uniform disclosure form, all authors declare the following: Payment/services info: All authors have declared that no financial support was received from any organization for the submitted work. Financial relationships: All authors have declared that they have no financial relationships at present or within the previous three years with any organizations that might have an interest in the submitted work. Other relationships: All authors have declared that there are no other relationships or activities that could appear to have influenced the submitted work.

\section{References}

1. Demirci H, Christianson MD: Orbital and adnexal involvement in sarcoidosis: analysis of clinical features and systemic disease in 30 cases. Am J Ophthalmol. 2011, 151:1074-80.e1. 10.1016/j.ajo.2010.12.011

2. Vahdani K, Rose GE: Sarcoid-like granulomatous orbitopathy: presentation, systemic involvement and clinical outcome. Eye (Lond). 2021, 35:470-6. 10.1038/s41433-020-0874-4

3. De Conti ML, Osaki MH, Sant'Anna AE, Osaki TH: Orbitopalpebral and ocular sarcoidosis: what does the ophthalmologist need to know. Br J Ophthalmol. 2022, 106:156-64. 10.1136/bjophthalmol-2020-317423

4. Prabhakaran VC, Saeed P, Esmaeli B, et al.: Orbital and adnexal sarcoidosis. Arch Ophthalmol. 2007, 125:1657-62. 10.1001/archopht.125.12.1657

5. Shinder R, Al-Zubidi N, Esmaeli B: Survey of orbital tumors at a comprehensive cancer center in the United States. Head Neck. 2011, 33:610-4. 10.1002/hed.21498

6. Mavrikakis I, Rootman J: Diverse clinical presentations of orbital sarcoid . Am J Ophthalmol. 2007, 144:76975. 10.1016/j.ajo.2007.07.019

7. Segal EI, Tang RA, Lee AG, Roberts DL, Campbell GA: Orbital apex lesion as the presenting manifestation of sarcoidosis. J Neuroophthalmol. 2000, 20:156-8. 10.1097/00041327-200020030-00003

8. Frohman LP, Guirgis M, Turbin RE, Bielory L: Sarcoidosis of the anterior visual pathway: 24 new cases . J Neuroophthalmol. 2003, 23:190-7. 10.1097/00041327-200309000-00002

9. Leclercq M, Sené T, Chapelon-Abric C, et al.: Prognosis factors and outcomes of neuro-ophthalmologic sarcoidosis [PREPRINT]. Ocul Immunol Inflamm. 2020, 1-8. 10.1080/09273948.2020.1834585

10. Kaeser PF, Borruat FX: Extraconal orbital sarcoidosis: an unusual cause of optic neuropathy . Klin Monbl Augenheilkd. 2010, 227:330-1. 10.1055/s-0029-1245211

11. Ing EB, Garrity JA, Cross SA, Ebersold MJ: Sarcoid masquerading as optic nerve sheath meningioma . Mayo Clin Proc. 1997, 72:38-43. 10.4065/72.1.38

12. Japanese Society of Sarcoidosis and Other Granulomatous Disorders: Diagnostic criteria of sarcoidosis 2015 . (2015). Accessed: January 14, 2022: https://www.jssog.com/wp/wpcontent/themes/jssog/images/system/guidance/1-2.pdf.

13. Patel BC, De Jesus O, Margolin E: Optic nerve sheath meningioma. StatPearls [Internet]. StatPearls Publishing, Treasure Island (FL); 2021.

14. Xie JS, Donaldson L, Margolin E: Optic perineuritis: a Canadian case series and literature review . J Neurol Sci. 2021, 430:120035. 10.1016/j.jns.2021.120035 